\title{
Framelet analysis of some geometrical illusions
}

\author{
Hitoshi Arai · Shinobu Arai
}

Received: 4 February 2010 / Revised: 14 February 2010 / Published online: 3 June 2010

(C) The Author(s) 2010

\begin{abstract}
In this paper we study a spiral illusion generated by fractal islands. Furthermore, by a neuro-scientific consideration we present a new class of geometrical illusions. In order to analyse these illusions, we propose a new mathematical method.
\end{abstract}

Keywords Geometrical illusion - Wavelet frame - Framelet - Extrastriate visual cortex

Mathematics Subject Classification (2000) $\quad 92 \mathrm{C} 99 \cdot 98 \mathrm{~A} 08 \cdot 68 \mathrm{U} 10$

\section{Introduction}

We often perceive images which are different from real objects. This phenomenon is called visual illusion or optical illusion. There are several types of visual illusions. In this paper we will study two types of geometrical illusions. One of them is the fractal spiral illusion which we made in 2007. Another is a new class of geometrical illusions which we introduce in this paper. We call them hyperbolic illusions. In Sect. 2 we will study the fractal spiral illusion by using wavelet-like filters. Section 3 is concerned with hyperbolic illusions and with mathematical analysis of them. In Sect. 4 we will

H. Arai was supported partly by Precursory Research for Embryonic Science and Technology, Japan Science and Technology Agency, and by Grant-in-Aid for Scientific Research (B), Japan Society for the Promotion of Science.

\section{H. Arai (凶)}

Graduate School of Mathematical Sciences, University of Tokyo, 3-8-1 Komaba,

Meguro-ku, Tokyo 153-8914, Japan

e-mail: h-arai@ms.u-tokyo.ac.jp

S. Arai

Bunkyo-ku, Tokyo, Japan 
present miscellaneous results. In Sect. 5 we will give a mathematical description of the method of our analysis. The last section is concerned with further problems.

A part of results on the fractal spiral illusion stated in Sect. 2 was announced briefly in Grant-in-Aid for Scientific Research News (Kakenhi News) 1 (2009), p. 8 without description of our method.

\section{Fractal spiral illusion}

In 1908, Fraser [9] discovered a spiral illusion which is now called Fraser's spiral illusion (see Fig. 1). In Fig. 1 the reader may perceive a spiral of a cord mixed black and white. However the cord is actually concentric circles. This illusion is very famous as a geometrical illusion. Recently Kitaoka et al. [15] have thrown new light on the spiral illusion. As we will mention later, in [15] they proposed a principle of making spiral illusions, and gave several examples of spiral illusions. In 2007 we found a new spiral illusion called a fractal spiral illusion or a fractal spiral figure (Fig. 2), which has greater illusion magnitude. It consists of copies with various sizes of a well known self-similar set called the fractal island. When we see the fractal spiral figure, we perceive that fractal islands form an anti-clockwise spiral. However actually the islands are arrayed like concentric circles.

In this section we will give a mathematical analysis of the fractal spiral illusion.

\subsection{Method}

Before stating our results we mention very briefly our mathematical method of analysing the fractal spiral illusion. Detailed description will be presented in Sect. 5. Our method is based on two tools: one of them is 'geometric filtering' which is introduced in this paper, and another is a simple pinwheel framelet constructed in Arai and

Fig. 1 Fraser's spiral illusion

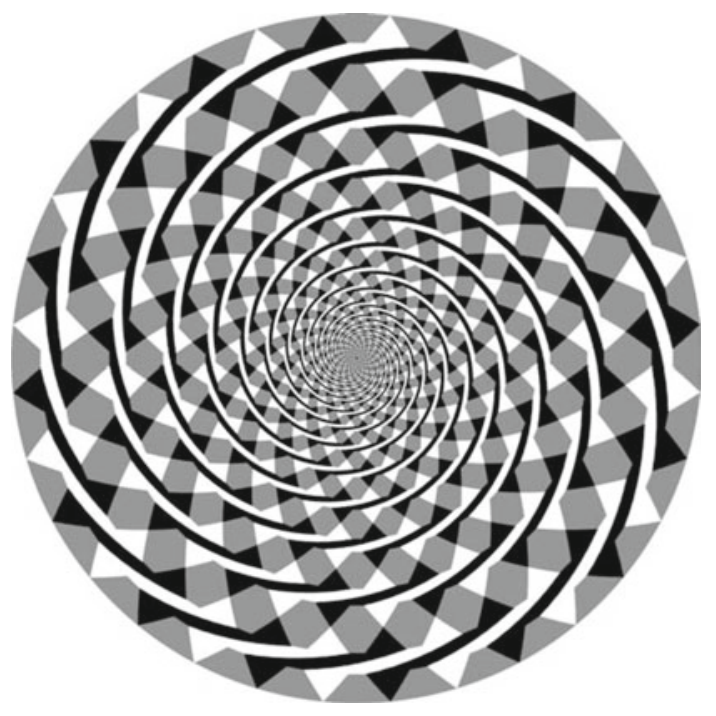




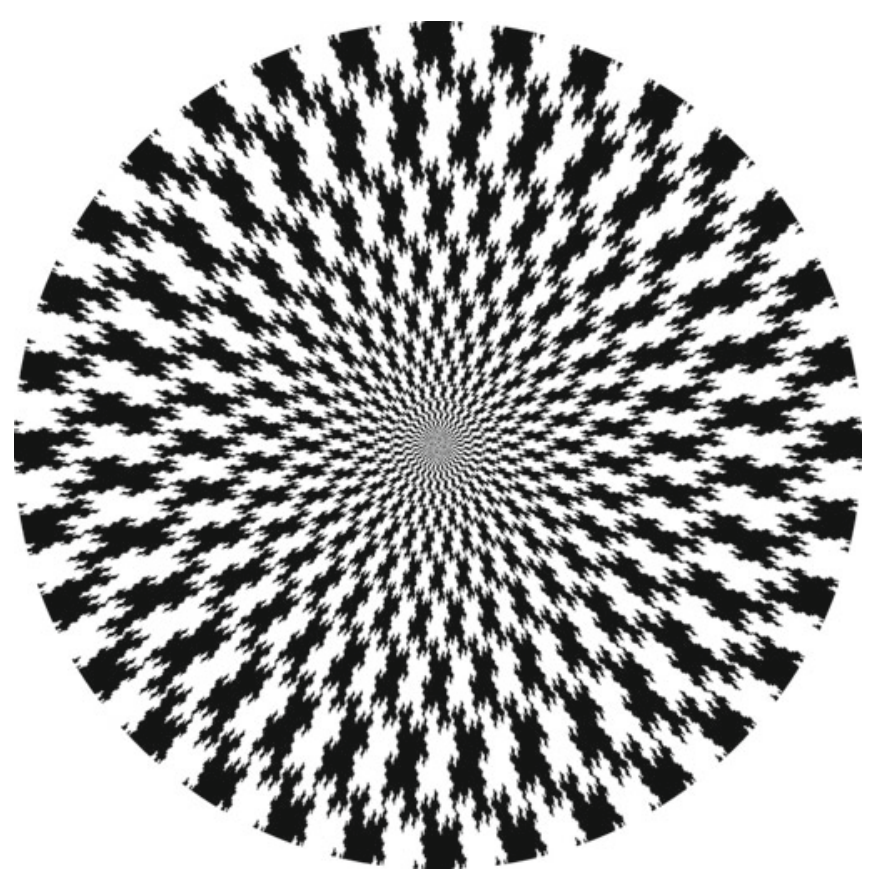

Fig. 2 The fractal spiral illusion (Arai and Arai, 2007, unpublished, see Remark 1)
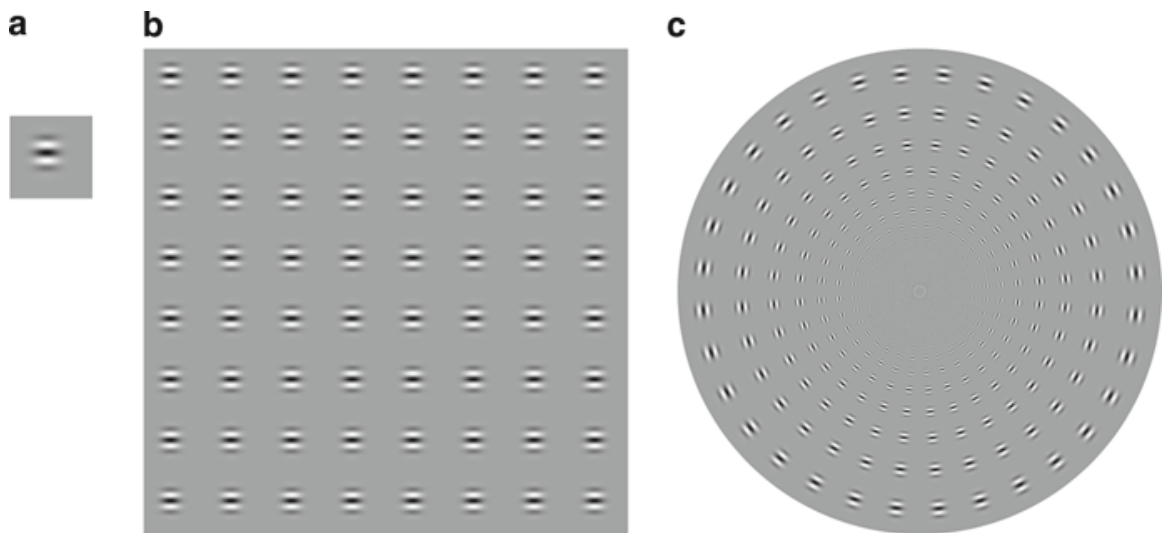

Fig. 3 a A filter in the 4th level of MOGMRA by the simple pinwheel framelet of degree 7; $\mathbf{b}$ a schematic figure of parallel moving of a filter shown in a. This is related to usual filtering (convolution); $\mathbf{c}$ a schematic figure of the movement of the filter related to concentric circular filtering

Arai [5]. We will start with 'geometric filtering'. The usual filtering is executed by a convolution, that is, an input signal is multiplied by a filter which is moved in parallel. Figure $3 \mathrm{~b}$ is a schematic figure representing parallel movement of the filter shown in Fig. 3a. On the other hand, a geometrical filtering is executed roughly speaking by moving a filter by a general geometrical rule. For example, filtering which we call 'concentric circular filtering' is based on the movement as in Fig. 3c. We will describe the exact definition of the geometric filtering in Sect. 5. 


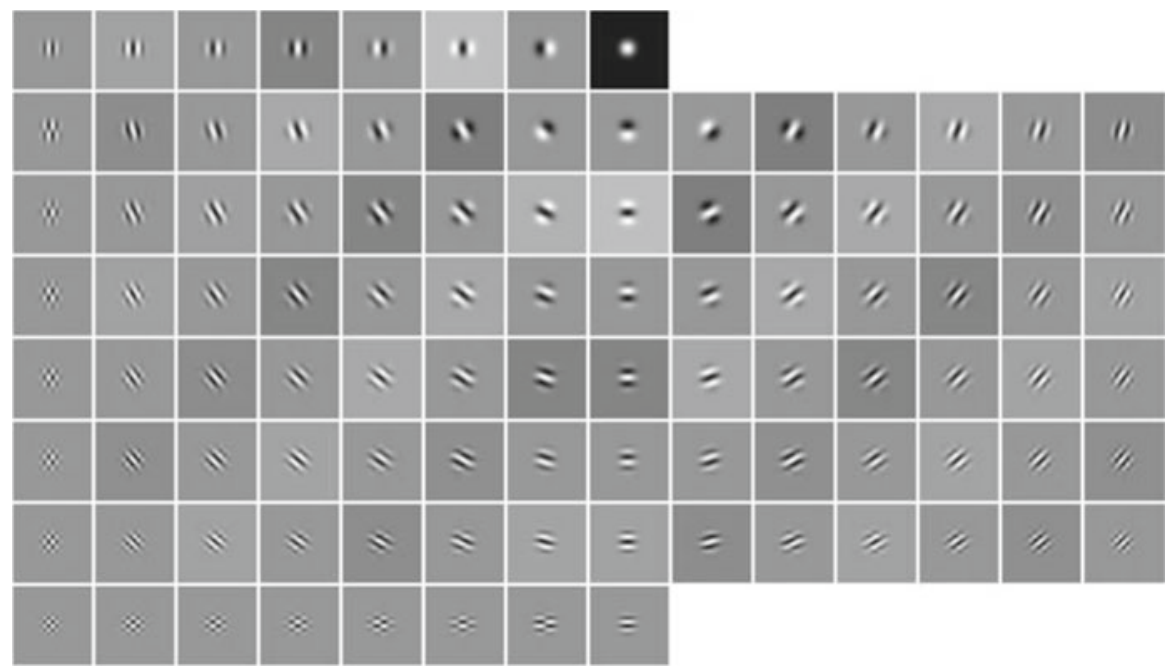

Fig. 4 Filters in the 2nd level of MOGMRA by the simple pinwheel framelet of degree 7: More precisely, using the notation stated in Sect. 5, they are decomposed approximation (top right) and decomposed details for a unit impulse

In this paper we apply the concentric circular filtering to filters in a maximal overlap, generalized multiresolution analysis (MOGMRA) based on a simple pinwheel framelet (see Arai-Arai [5] for the definition of MOGMRA and of the simple pinwheel framelet): First we explain MOGMRA. Originally maximal overlap, multiresolution analysis for a wavelet was established and studied by Coifman and Donoho [7], Nason and Silverman [18], and Percival and Walden [19]. A characteristic feature of MOGMRA is that MOGMRA produces multiple-level decomposition of an input signal, as in the case of the maximal overlap, multiresolution analysis for a wavelet. To put it briefly, the lower the level is, the more the resolution of the decomposed signal becomes down. In this paper we take 5 as the total number of levels. Next we turn to simple pinwheel framelets. The general theory of framelets was originated by Daubechies et al. [8]. We constructed simple pinwheel framelets for modelling visual information processing by simple cells in V1: They are filter banks which contain several filters having the various orientation selectivity. Moreover our framelets are equipped with a parameter called 'degree' with which we can control performance of orientation selectivity. The larger the parameter is, the more the performance of the orientation selectivity goes up. In this paper we set the degree to seven. For example, we show filters in the second level of MOGMRA by the simple pinwheel framelet of the degree 7 in Fig. 4. As shown in Fig. 4, each level of MOGMRA by the simple pinwheel framelet consists of one scaling filter at that level (top right) and 99 framelet filters. Since the total number of levels is 5, MOGMRA produces 496 decomposed signals: one is obtained by using the scaling filter at the 5 th level, and $99 \times 5$ signals are produced by using framelet filters. Let $X$ be the fractal spiral figure. Now by concentric circular filtering with respect to these filters we obtain also 496 decomposed signals of $X$ : one is related to the scaling filter at the 5 th level and others are related to framelet filters. We denote them by $R_{0}$ and $R_{1}, \ldots, R_{495}$, respectively. Since simple 

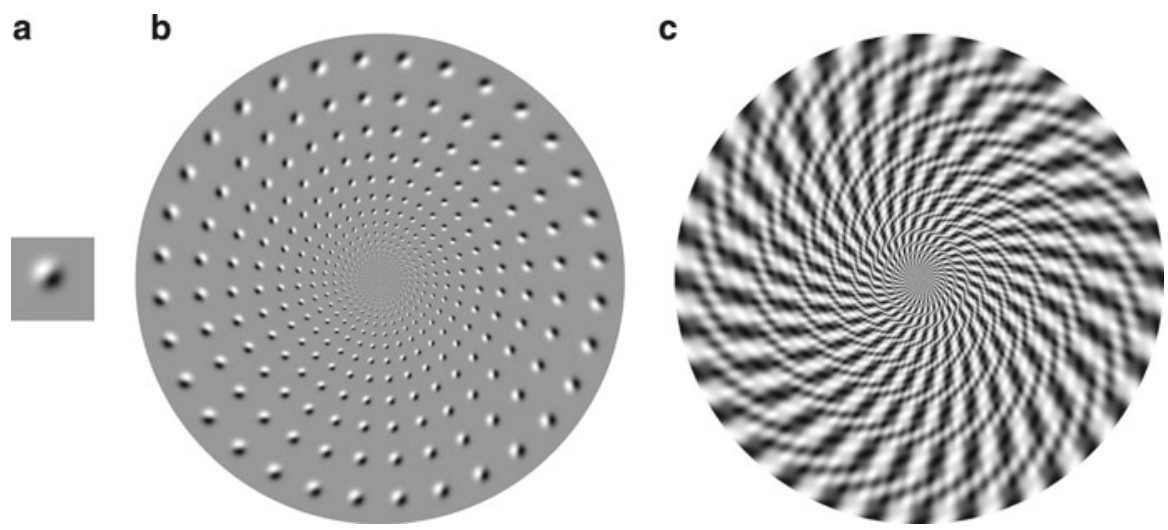

Fig. 5 a A filter in the 4th level of MOGMRA by the simple pinwheel framelet of degree 7; $\mathbf{b}$ a schematic figure of concentric circular filtering by the filter shown in $\mathbf{a}$; $\mathbf{c}$ the CMRA reconstructed detail for the fractal spiral figure with respect to the filter in $\mathbf{a}$. This is of type A

pinwheel framelets have the perfect reconstruction property [5], we have that

$$
X=R_{0}+R_{1}+\cdots+R_{495}
$$

We call this decomposition the circular filtering, maximal overlap, multiresolution analysis (abbrev. CMRA). We call each $R_{j}$ for $j \geqq 1$ a CMRA reconstructed detail, and $R_{0}$ the CMRA reconstructed approximation at the 5th level. In this section we consider as an input signal the fractal spiral illusion.

\subsection{Results}

As warm-ups, we show 4 typical examples of CMRA reconstructed details for the fractal spiral figure:

Example 1 (Type A) Take a framelet filter shown in Fig. 5a from the simple pinwheel framelet. Figure $5 \mathrm{~b}$ is a schematic figure representing the concentric circular filtering by the framelet filter. The CMRA reconstructed detail with respect to the filter is shown in Fig. 5c. We should note that we can perceive an anti-clockwise spiral illusion in Fig. 5c. For convenience we call such CMRA reconstructed detail a type A signal.

Example 2 (Type N) Here we deal with a framelet filter shown in Fig. 6a. Figure 6b is a schematic figure of concentric circular filtering corresponding to the filter in Fig. 6a. The CMRA reconstructed detail is Fig. 6c. As opposed to type A signals, we can perceive no spiral illusions in Fig. 6c. We call such CMRA reconstructed details type $\mathrm{N}$ signals.

Example 3 (Type $C$ ) Surprisingly we can find CMRA reconstructed details which provide us clockwise spiral illusions (see Fig. 7c). We call such CMRA reconstructed details type $\mathrm{C}$ signals. 

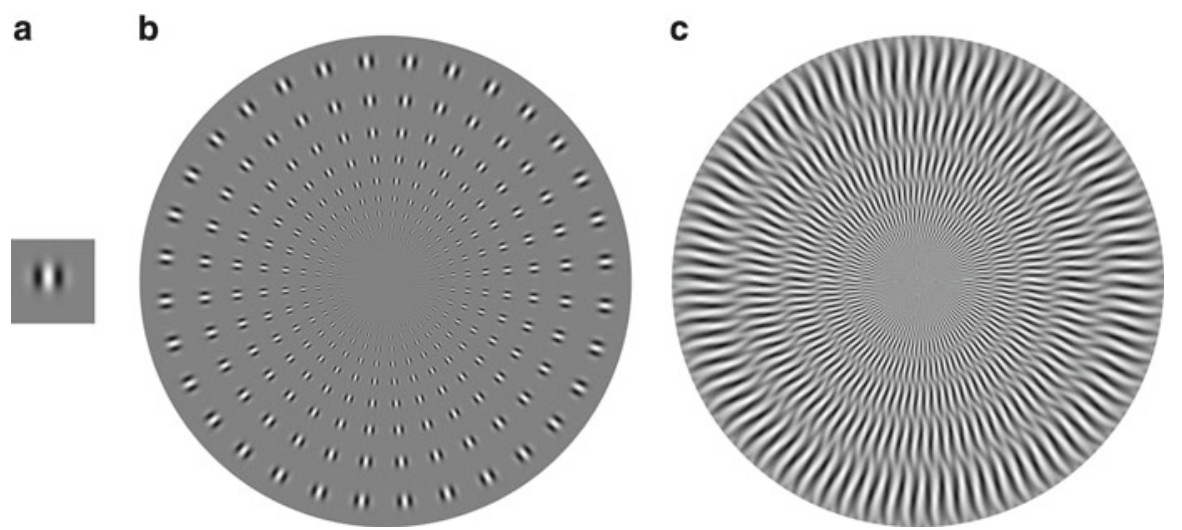

Fig. 6 a A filter in the 4th level of MOGMRA by the simple pinwheel framelet of degree 7; $\mathbf{b}$ a schematic figure of concentric circular filtering by the filter shown in $\mathbf{a}$; $\mathbf{c}$ the CMRA reconstructed detail for the fractal spiral figure with respect to the filter in a. This is of type $\mathrm{N}$
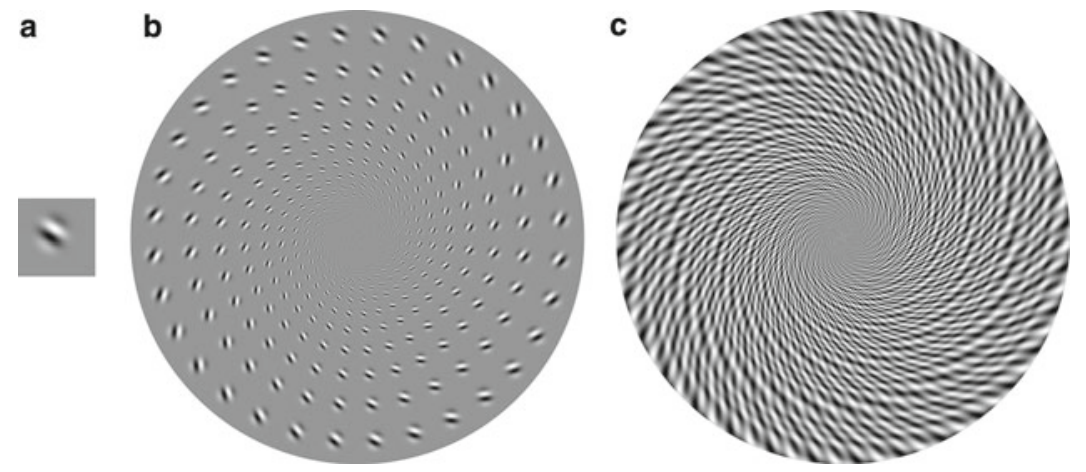

Fig. 7 a A filter in the 4th level of MOGMRA by the simple pinwheel framelet of degree 7; $\mathbf{b}$ a schematic figure of concentric circular filtering by the filter shown in a; $\mathbf{c}$ the CMRA reconstructed detail for the fractal spiral figure with respect to the filter in a. This is of type $\mathrm{C}$
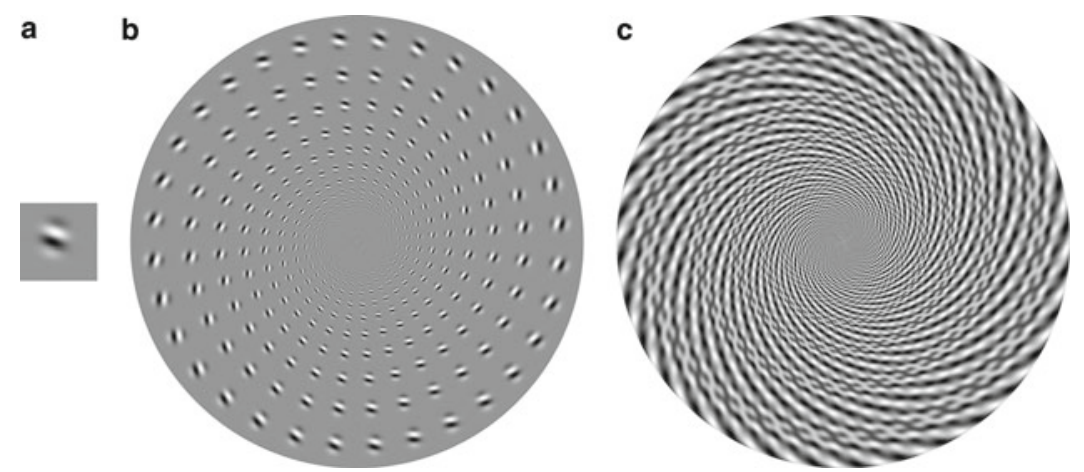

Fig. 8 a A filter in the 4th level of MOGMRA by the simple pinwheel framelet of degree 7; $\mathbf{b}$ a schematic figure of concentric circular filtering by the filter shown in a; $\mathbf{c}$ the CMRA reconstructed detail for the fractal spiral figure with respect to the filter in a. This is of type AC 


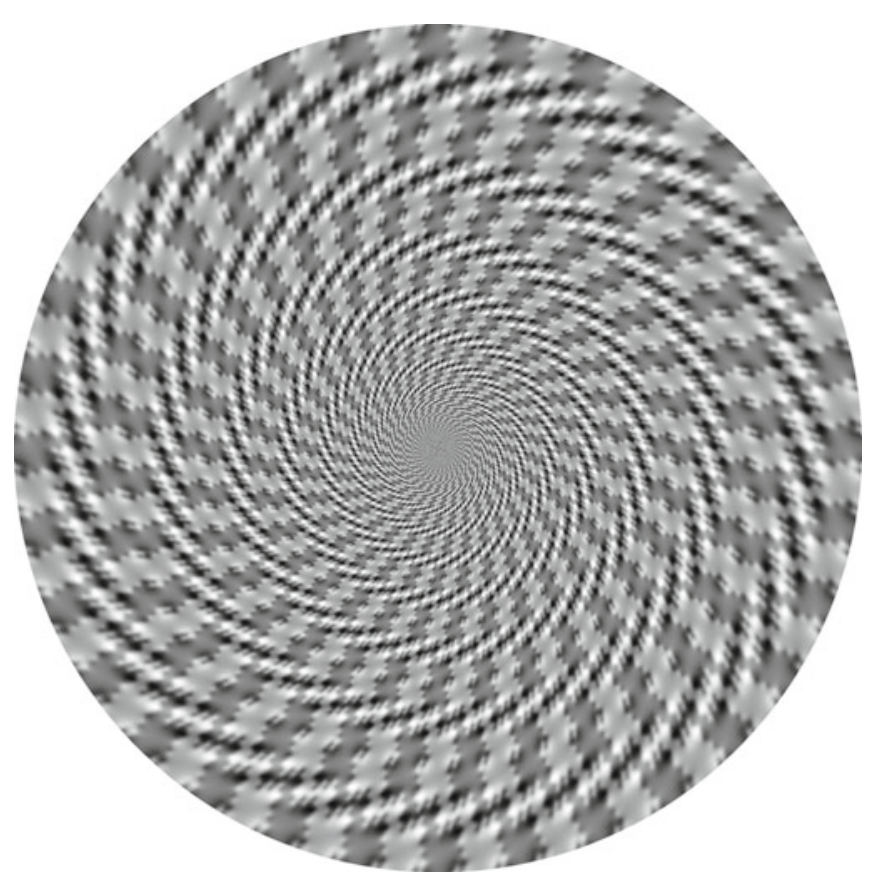

Fig. $9 X_{A}$. This is regarded as anti-clockwise illusory component of the fractal spiral illusion. We can find in this image curved twisted type cords. These are concentric circles, but apparently spiral. Compare the classical Fraser's spiral illusion (Fig. 1)

Example 4 (Type AC) We have also CMRA reconstructed details which give us both anti-clockwise and clockwise spiral illusions (see Fig. 8c). We call such CMRA reconstructed details type AC signals.

Experimentally we can sort all CMRA reconstructed details into the above mentioned types A, N, C, and AC. Denote by $X_{A}$ the sum of all type A signals. By the same way we can define also $X_{N}, X_{C}$, and $X_{A C}$. An important property of these signals is that the original signal $X$, that is, the fractal spiral figure, is equal to the sum of them and $R_{0}$ :

$$
X=R_{0}+X_{A}+X_{N}+X_{C}+X_{A C}
$$

This equality is easily proved by (1).

The pictures of the signals $X_{A}, X_{C}$, and $X-X_{A}-X_{C}-X_{A C}$ are Figs. 9, 10, and 11 , respectively.

\subsection{Discussion on the fractal spiral illusion}

In their paper [15], Kitaoka et al. discovered that spiral illusions are induced by transforming segments that produce a tilt illusion to concentric circles, and they infer also 


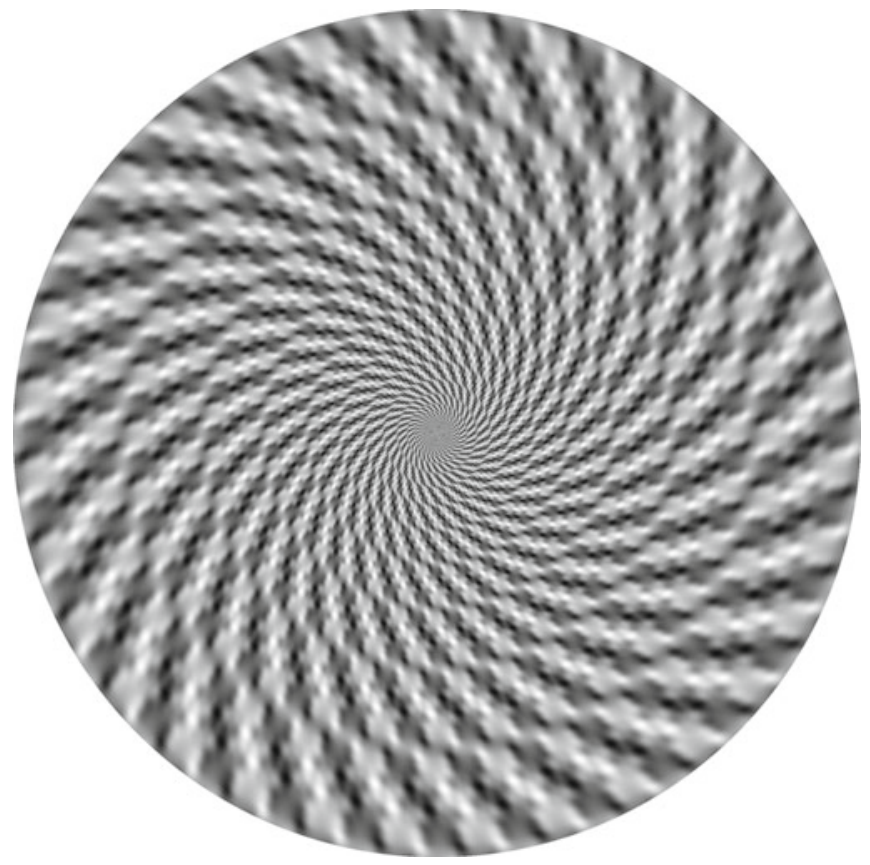

Fig. $10 X_{C}$. Clockwise illusory component of the fractal spiral illusion

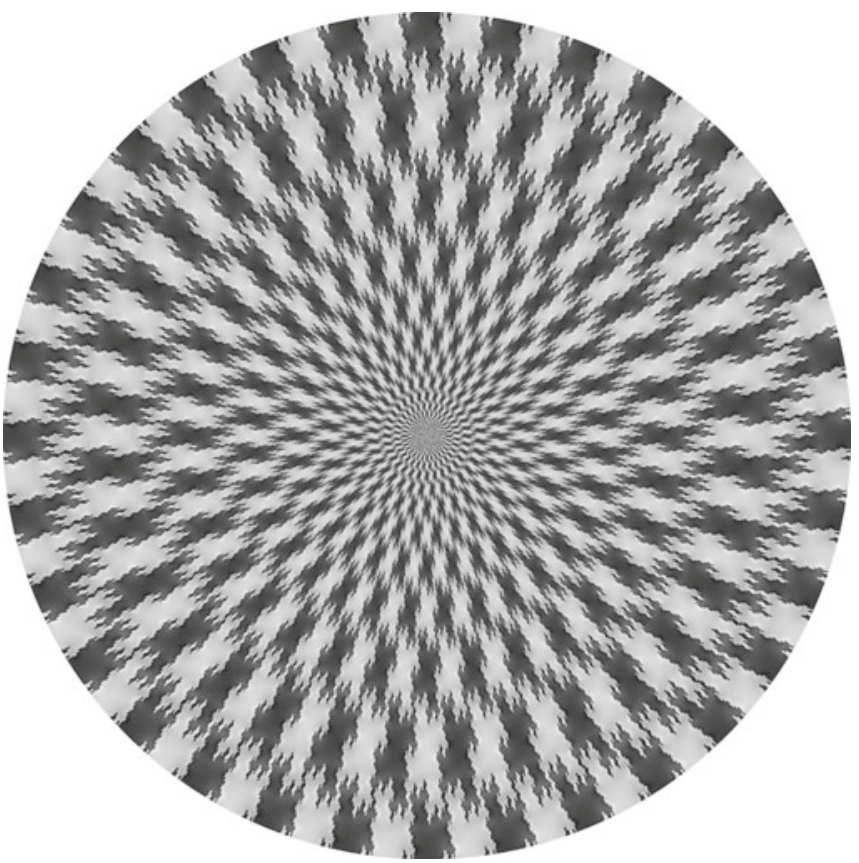

Fig. $11 X-X_{A}-X_{C}-X_{A C}$. Removal of illusory components from the fractal spiral illusion 
Fig. $12 X-X_{C}$. Removal of clockwise illusory components strengthens the anti-clockwise illusion magnitude of the fractal spiral figure. Compare this figure with the original fractal spiral illusion (Fig. 2)

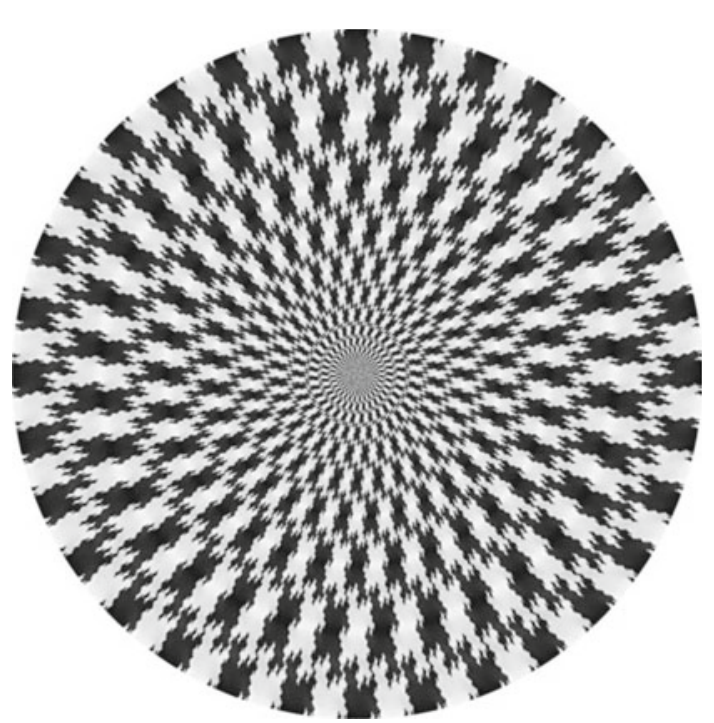

that "the spiral effect is a consequence of a mechanism that fires a unitary spiral detector". As pointed out in Gallant et al. [10,11], the area V4 of the visual cortex contains neurons which are highly selective for Cartesian, polar, and hyperbolic grating (for simplicity we call them neurons of Cartesian type, of polar type and of hyperbolic type). In particular Gallant et al. found that some neurons in V4 fire strongly to concentric circles, to spirals, and to radial patterns. Kitaoka et al. [15] asserted "this finding adds plausibility to the existence of the detector of spirals". We think that the concentric circular filterings with respect to a maximal overlap, simple pinwheel framelet provide a mathematical representation of the information processing by such V4 neurons.

By the result of our mathematical analysis mentioned in the previous section, we can say that the structure of the illusion of the fractal spiral figure is not simple. Indeed, some filters generate illusory signals (anti-clockwise spiral illusion), however some filters produce clockwise spiral illusions although we perceive anti-clockwise illusion in the fractal spiral figure. If it is true that the concentric circular filterings represent mathematically the processing by V4 neurons, our computer experiment implies that some of V4 neurons trigger the fractal spiral illusion, and some of V4 neurons produce a factor of the opposite illusion. However the latter factor does not attain to the perception of opposite illusions. Now it seems to be natural to think that the outputs of the latter neurons inhibit the occurrence of the fractal spiral illusion. In order to investigate this speculation, we extract all type $\mathrm{C}$ signals from the original signal $X$. Then we gain Fig. 12. As we expected, the illusion magnitude of Fig. 12 is stronger than one of the original fractal spiral figure (Fig. 2). This means that type $\mathrm{C}$ signals certainly inhibit the fractal spiral illusion.

Lastly we note that by the same method we can remove illusory component from the classical Fraser's spiral illusion.

We think that our mathematical method is completely new, and that it has developed the study of spiral illusions. 
Fig. 13 Fraser illusion or

Fraser's twisted cords on

chequer-work background

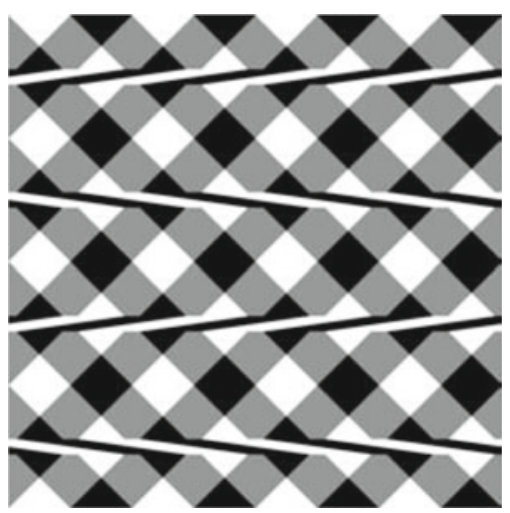

\section{Hyperbolic illusions}

In this section, we generate new geometrical illusions from tilt illusions, and study them by mathematical method like the previous section.

\subsection{Motivation}

As Gallant et al. revealed, V4 contains neurons of Cartesian type, polar type, and hyperbolic type. Given that neurons of polar type induce spiral illusions [15], the following questions arise naturally: Are there visual illusions related to neurons of other types? And what kinds of illusions are they? One can easily consider that tilt illusions are related to neurons of Cartesian type. Now what happens in the case of hyperbolic type? This is the motivation of this section.

\subsection{Preliminary observation}

Let us begin with going back to Fraser's work [9]. Fraser found a famous tilt illusion which is called now by Fraser illusion or Fraser's 'twisted cord on chequer-work background' illusion (see Fig. 13). Figure 13 has two types of horizontal cords. One of them is the top cord and the third cord from the top: these appear to tilt anti-clockwise. Indeed, for example the positive angle from the top cord to the vertical axis seems to be contracted. Another is the second and fourth cords from the top that appear to tilt clockwise. The positive angle from the second (or fourth) cord to the vertical axis seems to be expanded. Fraser's spiral illusion is essentially made by concentrically circling Fraser's twisted cords: if one uses cords which produce anti-clockwise tilt illusion, one obtains Fig.1, and if one uses twisted cords which produce clockwise tilt illusion, one has a figure which gives us the impression that concentric circled cords form clockwise spirals. Kitaoka et al. [15] showed experimentally that such deformation of any tilt illusions induces spiral illusions.

It is interesting to note that these phenomenon we have described indicate that apparent angles are 'approximately' preserved under the conformal mapping which transforms Cartesian coordinate axes to polar ones. Here and throughout this paper, 

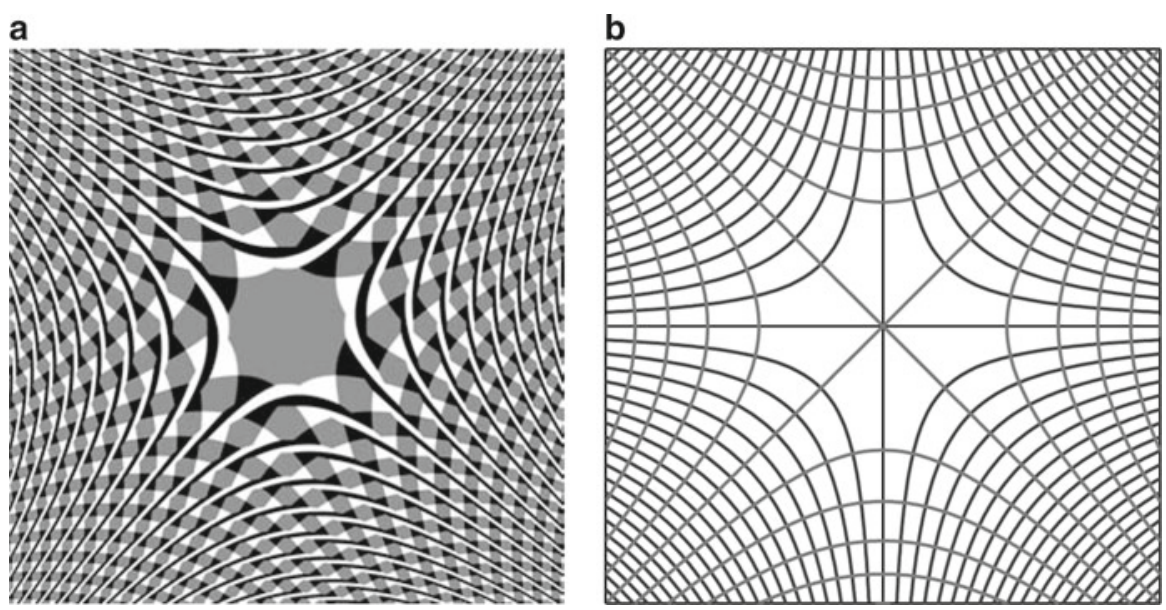

Fig. 14 a Hyperbolic Fraser illusion. Bended twisted cords which are arrayed along rectangular hyperbolic coordinate axes seem to tilt anti-clockwise; $\mathbf{b}$ the rectangular hyperbolic coordinate

'approximately' preserving under a mapping means that the occurrence of illusions and also orientations of apparent angles are preserved; it does not mean that apparent angles are completely preserved.

\subsection{Results}

\subsubsection{Hyperbolic illusions}

Let us now return to our problem. Both the existence of neurons of hyperbolic type and the observation in the previous subsection lead us to the following conjecture: apparent angles will be 'approximately' preserved under the conformal mapping which transforms Cartesian coordinate axes to rectangular hyperbolic coordinate axes (see Sect. 3.2 for the definition of 'approximately' preserving). For convenience, we call such a mapping the hyperbolic conformal mapping. If the conjecture is correct, then bended twisted cords along the rectangular coordinate axes must apparently slant from the corresponding axes. Namely an illusion occurs. In order to investigate this observation, we make such a figure. As we will describe in Sect. 5, we deform twisted cords by using the hyperbolic conformal mapping. The result is Fig. 14a. Although the deformed twisted cords are arrayed along the rectangular hyperbolic coordinate axes, we perceive that they slant anti-clockwise from diagonal axes. This can be confirmed by comparing Fig. 14a with the rectangular hyperbolic coordinate (Fig. 14b). By the similar way, we can make also a figure such that the bended twisted cords seem to tilt from the horizontal axes and vertical ones. We call these new illusions the hyperbolic Fraser illusions.

We checked experimentally also that same phenomenon happens for other several tilt illusions. We call them hyperbolic illusions. 


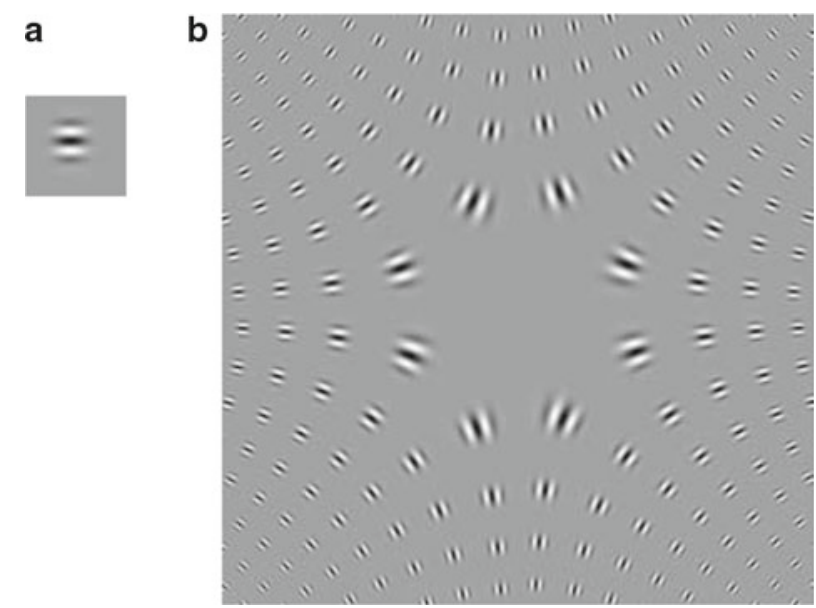

Fig. 15 a A filter in the 4th level of MOGMRA by the simple pinwheel framelet of degree 7; $\mathbf{b}$ a schematic figure of the movement related to the hyperbolic filtering

\subsubsection{Framelet analysis of hyperbolic illusions}

In order to analyse these illusions, we consider a geometrical filtering related to the hyperbolic conformal mapping, and call it the hyperbolic filtering. A schematic figure of the hyperbolic filtering is shown in Fig. 15 (for details, see Sect. 5). Based on the hyperbolic filtering, we can construct hyperbolic filtering version of MOGMRA from simple pinwheel framelets (abbrev. HMRA) by using the same idea as the case of CMRA. In this paper we use HMRA with five levels constructed by the simple pinwheel framelet of degree 7. By the same way as the CMRA case, we can define HMRA reconstructed details $R_{1}^{\prime}, \ldots, R_{495}^{\prime}$, and HMRA reconstructed approximation at the 5 th level $R_{0}^{\prime}$. Denote the HMRA decomposition of a signal $X$ by

$$
X=R_{0}^{\prime}+R_{1}^{\prime}+\cdots+R_{495}^{\prime}
$$

Using HMRA we study hyperbolic illusions, and as in the case of the fractal spiral illusion, we will extract illusory components from hyperbolic illusions.

In this paper we will concentrate our attention to a hyperbolic illusion generated by so-called fringed edges. The pattern of 'fringed edges' was found in Kitaoka et al. [15] (see also [14] and [13]). Kitaoka et al. created a tilt illusion by using fringed edges (see Fig. 16). Our hyperbolic illusion is Fig. 17. It consists of bended bands which are made by connecting in series the 'fringed edges' pattern. We call Fig. 17 the hyperbolic illusion of fringed edges. An interesting nature of this illusion is that bands of fringed edges not only along diagonal axes but also along horizontal axes and vertical axes slant anti-clockwise. The cause of this phenomenon is that the illusion of fringed edges has tilt illusions of both horizontal and vertical directions. Let $X$ be the hyperbolic illusion of fringed edges. We decompose the hyperbolic illusion of fringed edges by HMRA. Then we can classify experimentally all HMRA reconstructed details into the following types: 
Fig. 16 Illusion of fringed edges (or turtle) (Kitaoka et al. [15], see also [14])

Fig. 17 Hyperbolic illusion of fringed edges
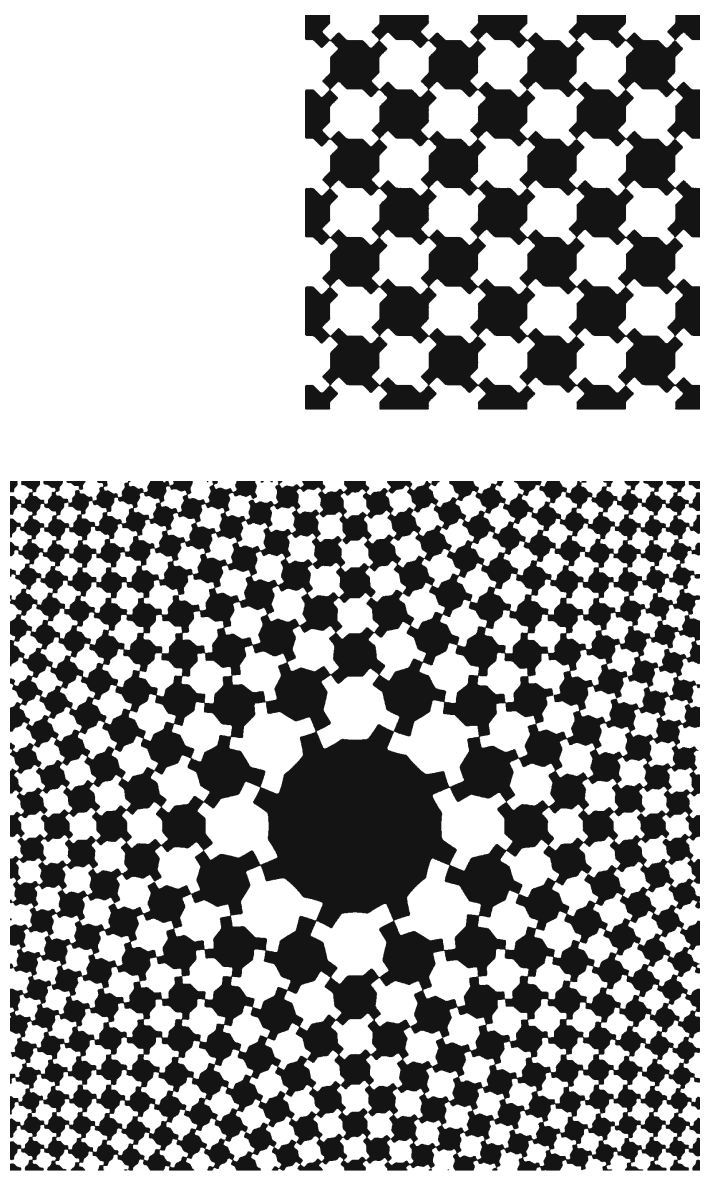

- Type DA: HMRA reconstructed details which provide us an illusion that bended bands slant off from the diagonal axes anti-clockwise.

- Type DC: HMRA reconstructed details which provide us an illusion that bended bands slant off from the diagonal axes clockwise.

- Type DAC: HMRA reconstructed details which provide us both anti-clockwise and clockwise hyperbolic illusion with respect to diagonal axes.

- Type HVA: HMRA reconstructed details which provide us an illusion that bended bands slant off from the horizontal axes and the vertical one anti-clockwise.

- Type HVC: HMRA reconstructed details which provide us an illusion that bended bands slant off from the horizontal axes and the vertical one clockwise.

- Type HVAC: HMRA reconstructed details which provide us both anti-clockwise and clockwise hyperbolic illusion with respect to horizontal and vertical axes.

- Type N: HMRA reconstructed details which provide us no illusions.

Here we show in Fig. 18c an example of type DA signals. 

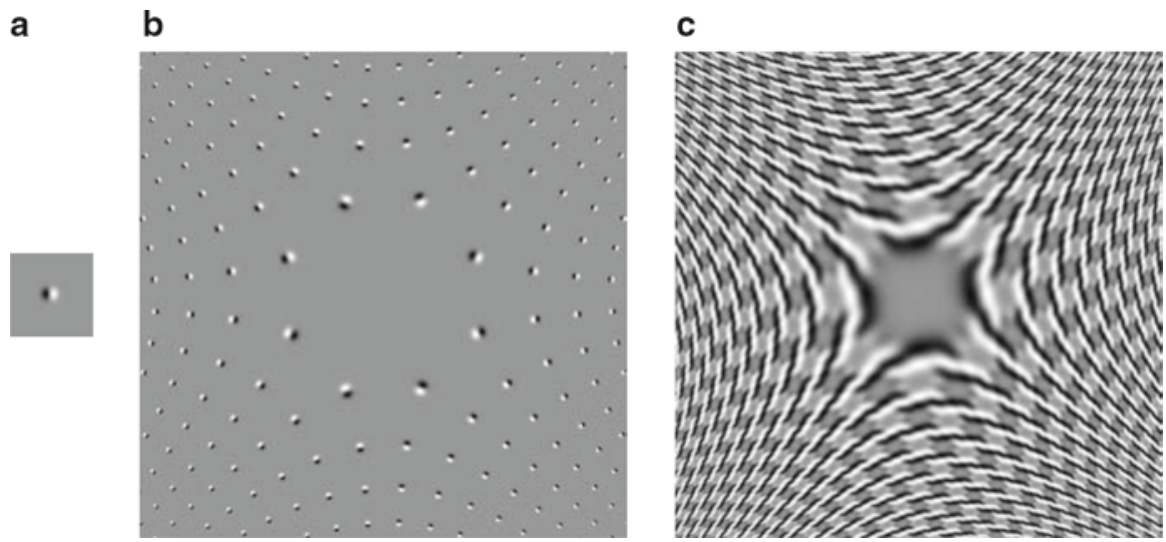

Fig. 18 a A filter in the 3rd level of MOGMRA by the simple pinwheel framelet of degree 7; $\mathbf{b}$ a schematic figure of 'hyperbolic movement' of the filter; c an example of type DA signal for the hyperbolic illusion of fringed edges

Fig. $19 X-X_{D A}-X_{D C}-$ $X_{D A C}-X_{H V A}-X_{H V C}-$ $X_{H V A C}$. Removal of illusory components from the hyperbolic illusion of fringed edges

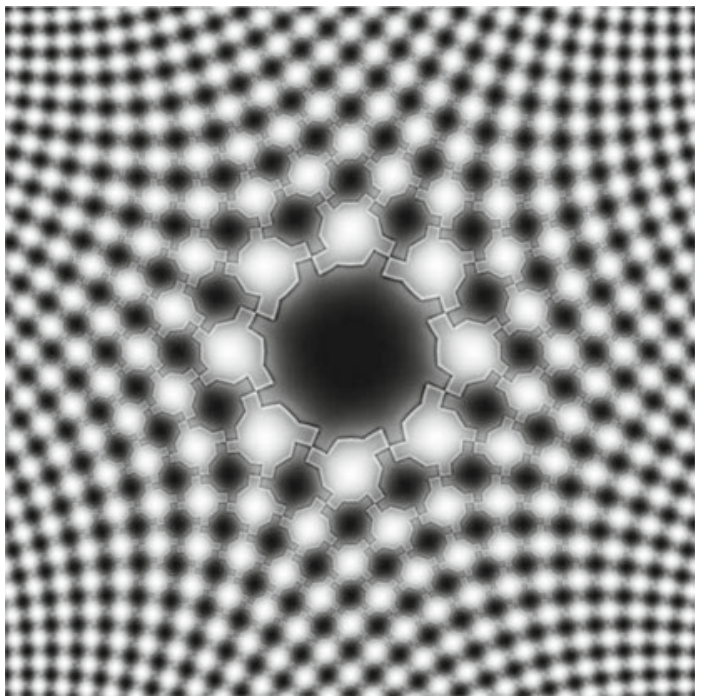

Let $X_{D A}$ be the sum of all type DA signals. By the same way we define $X_{D C}, X_{D A C}$, $X_{H V C}, X_{H V A}, X_{H V A C}$, and $X_{N}$. It should be noted that by the perfect reconstruction property of simple pinwheel framelets we have that

$$
X=R_{0}^{\prime}+X_{D A}+X_{D C}+X_{D A C}+X_{H V A}+X_{H V C}+X_{H V A C}+X_{N} .
$$

We show images of signals $X-X_{D A}-X_{D C}-X_{D A C}-X_{H V A}-X_{H V C}-X_{H V A C}$, $X-X_{H V A}-X_{H V C}-X_{H V A C}, X-X_{D A}-X_{D C}-X_{D A C}, X_{D A}, X_{H V A}$, and $X_{D A}+X_{H V A}$ in Figs. [19-24] respectively. We will explain these images in the next section. 
Fig. $20 X-X_{H V A}$

$-X_{H V C}-X_{H V A C}$. Removal of only vertical and horizontal illusion. The figure produces only diagonally illusion

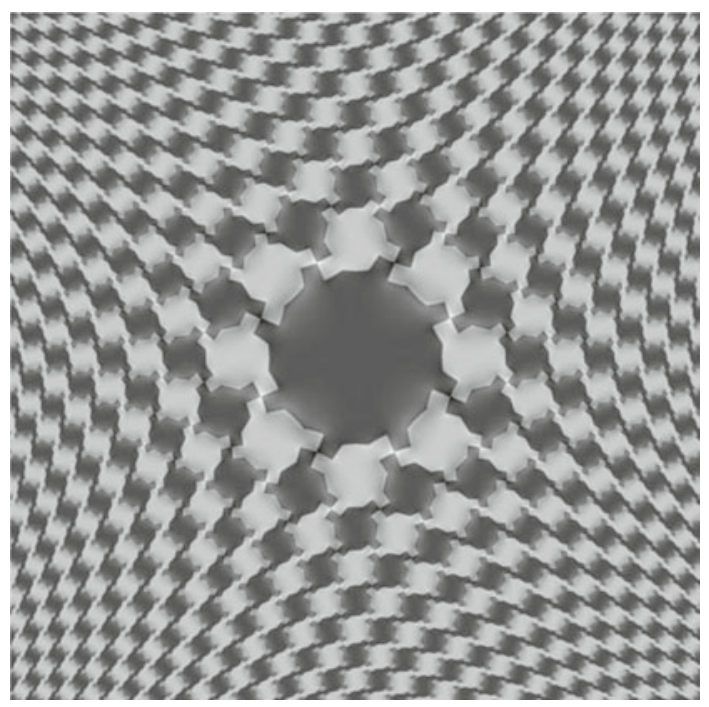

Fig. $21 X-X_{D A}-X_{D C}$ $-X_{D A C}$. Removal of only diagonal illusion. Horizontal and vertical illusions still remains

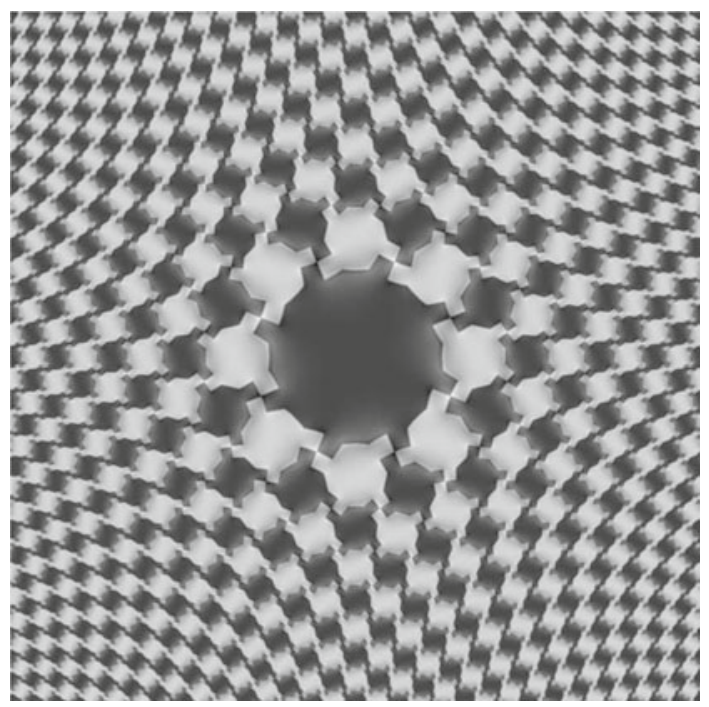

\subsection{Discussion on hyperbolic illusion of fringed edges}

An example of type DA signal appears in Fig. 18c. We can recognize in this figure cords similar to twisted cords of Fraser illusion. The signal $X_{D A}$ shown in Fig. 22 is the total sum of all type DA signals, and it also contains such cords. The signal $X_{H V A}$ similarly has twisted-like cords (see Fig. 23). In the case of Cafe Wall illusion [12], which is a tilt illusion, Morgan and Moulden showed that twisted-like cords appear by simple band-pass filtering [17]. In Arai and Arai [3] we showed that wavelet decompositions of many tilt illusions such as Cafe Wall illusion, illusion of fringed 
Fig. $22 X_{D A}$. Synthesized image by type DA signals, that is, diagonal anti-clockwise illusory component of the hyperbolic illusion of fringed edges

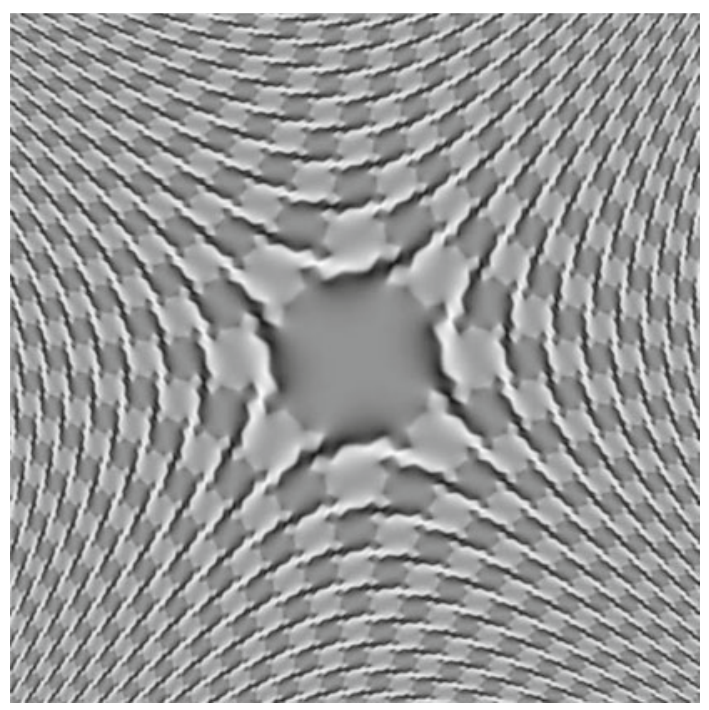

Fig. $23 X_{H V A}$. Synthesized image by type HVA signal, that is, horizontal and vertical anti-clockwise illusory component of the hyperbolic illusion of fringed edges

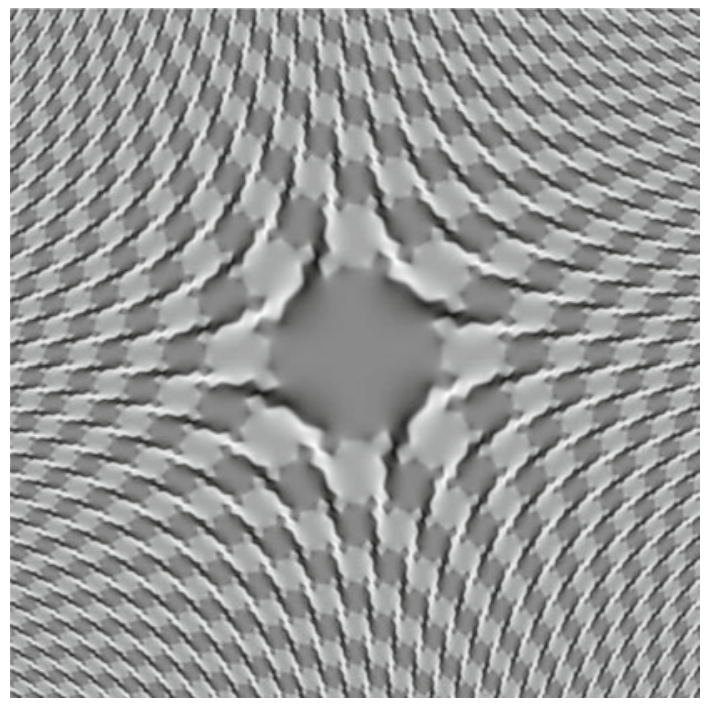

edges, etc. contain twisted-like cords. In that paper we used maximal overlap version of a biorthogonal wavelet constructed in Cohen et al. [6]. We refer [1] for nonlinear analysis of Cafe Wall illusion and twisted cords.

By our new mathematical method, we can identify 6 illusory components of the hyperbolic illusions of fringed edges: types DA, DC, DAC, HVA, HVC and HVAC. This classification permits us detailed analysis of the hyperbolic illusion. In fact we can handle accurately illusory components: For example, we extract the diagonal anti-clockwise illusory component (Fig. 22) or the horizontal and vertical anti-clockwise illusory component (Fig. 23) from the hyperbolic illusion of fringed edges. 
Fig. $24 X_{D A}+X_{H V A}$. This figure does not contain clockwise illusory components. Illusion magnitude of this figure is greater than one of the original hyperbolic illusion of fringed edges

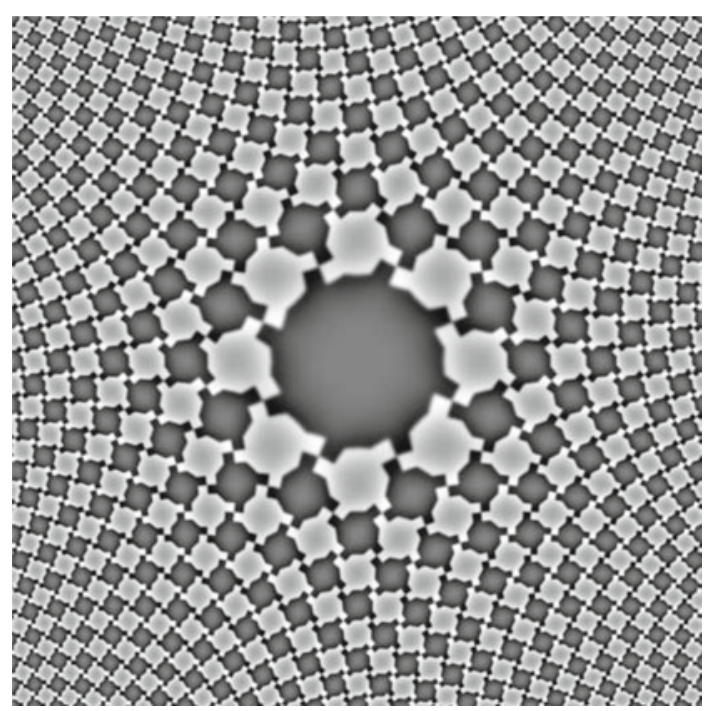

Moreover, we can eliminate all diagonal illusory components, that is, types DA, DC, and DAC signals, from the original figure. The result is shown in Fig. 21: we cannot perceive in this figure illusion along diagonal lines, but illusion along horizontal and vertical directions still remain. We can carry out the same analysis to horizontal-vertical illusory components. See Fig. 20 for the result. Figure 19 is the image extracted all illusory components, and we cannot see illusions in this image.

Figure 24 is the anti-clockwise illusory components $X_{D A}+X_{H V A}$. This does not contain clockwise illusory components, and consequently the illusion magnitude of Fig. 24 is greater than one of the original hyperbolic illusion of fringed edges.

\section{Miscellaneous results}

We found two interesting phenomena related to the fractal spiral illusion. In this section we report them. One of them is an effect to the fractal spiral illusion of coloring, and another is apparent distortion of concentric circles on the fractal spiral figure. First we show the effect of coloring. As we have seen in Fig. 2, the fractal spiral illusion is drawn in gray scale. We discovered that the occurrence of apparent spirals on the fractal spiral figure depends on coloring. In fact the following phenomena happens according to coloring: We have spiral illusory impression in Fig. 25 (top), but Fig. 25 (bottom) has no apparent spirals, although its design is completely same as the above figure. (Remark: Since the luminance of the bottom figure is almost uniform, in the print version it might be printed as a gray disc. Please see this figure in the online version.) The difference between two figures is that the luminance of Fig. 25 (top) is not uniformly, but one of Fig. 25 (bottom) is almost homogeneous. This property is a characteristic feature of the fractal spiral illusion. In fact, under the same luminance condition as Fig. 25 (bottom), Fraser' spiral illusion still remains (see Fig. 26). It should be noted that the above mentioned observation does not mean that the fractal spiral illusion disappear whenever the luminance is almost uniform. 
Fig. 25 Colored fractal spiral figure (top); colored fractal spiral figure with almost uniform luminance (bottom). In this case, the spiral illusion disappears. Remark: The luminance of the bottom figure is almost uniform. If it is printed not good in the print version, please see this figure in the online version
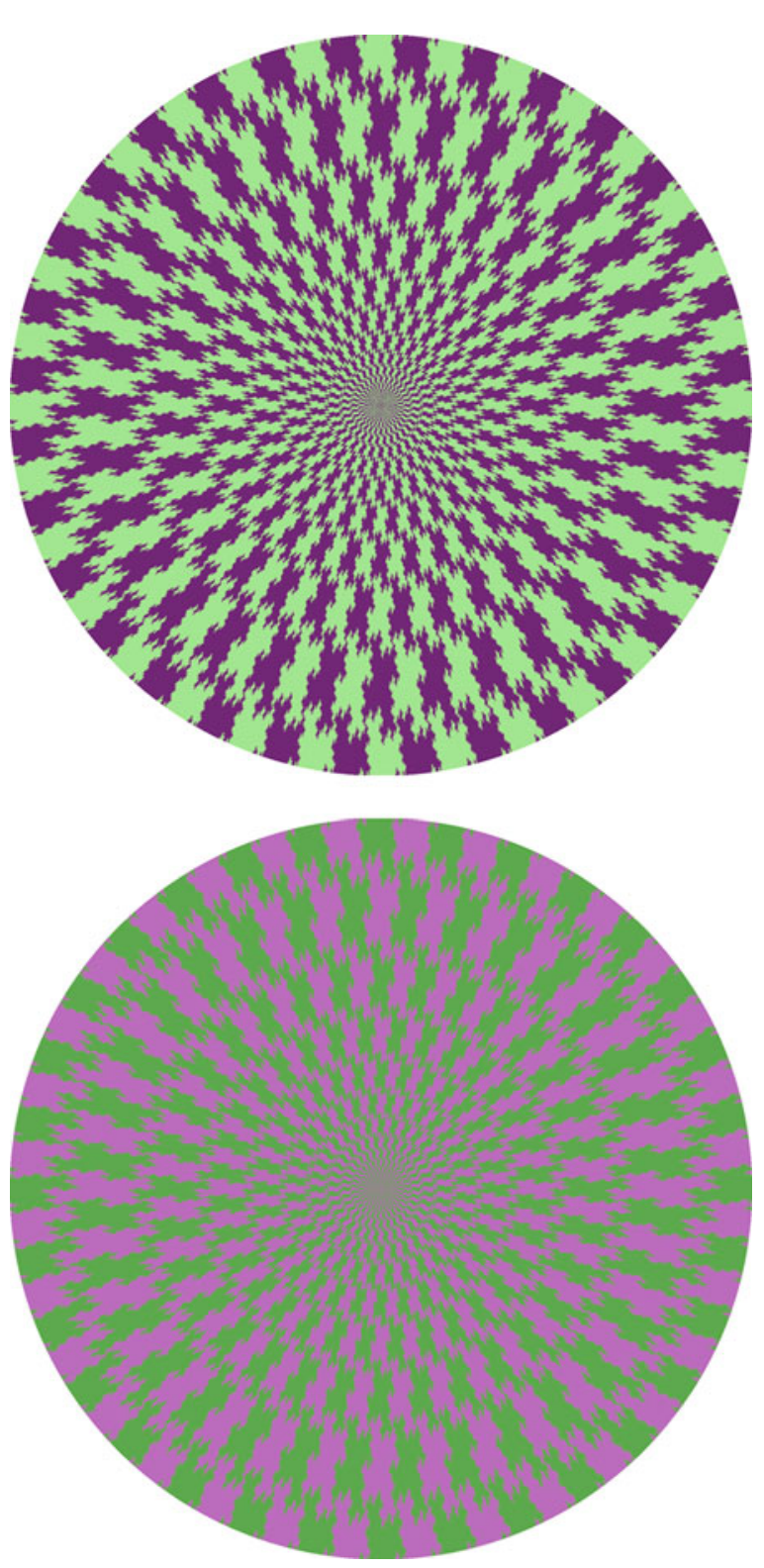

Next phenomenon is what we call the distorted circle illusion. In Fig. 27, green curves are actually concentric circles, but we perceive that the curves are distorted. J. Fraser showed in [9] illusions that some concentric circular, modified twisted cords seem to be warped. However there is a difference between Fraser's distorted circle illusions and ours. It is that the circles in Fig. 27 (top) is not twisted cords, but only circled lines. 
Fig. 26 Colored Fraser's spiral illusion with almost uniform luminance. In this case the illusion does not disappear. Remark: The luminance of the figure is almost uniform. If it is printed not good in the print version, please see this figure in the online version

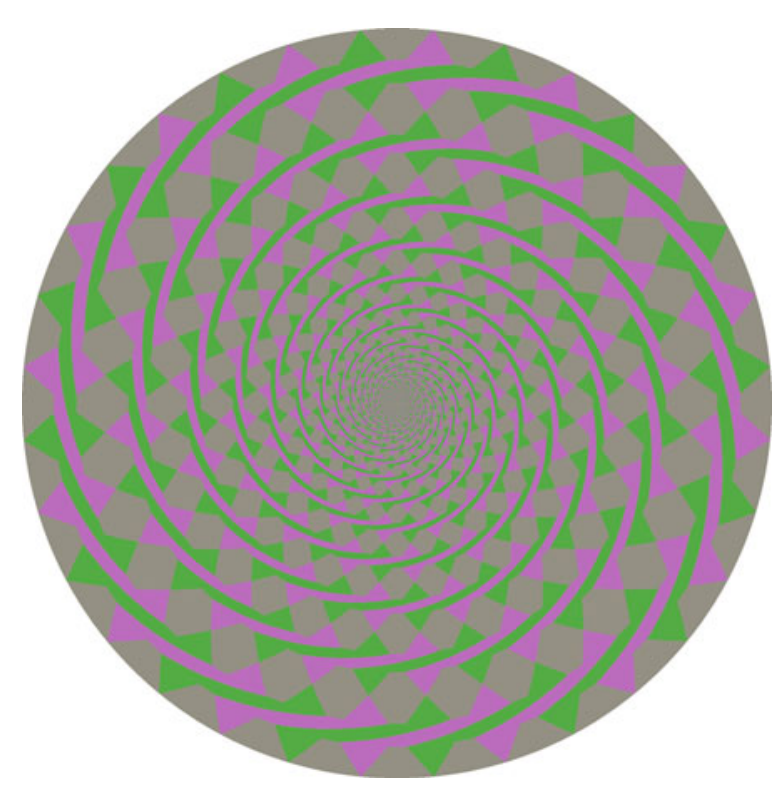

\section{Method of computation}

In this section we describe our method of computation used in Sects. 2 and 3. Let $x$ be an input signal to MOGMRA by simple pinwheel framelet of degree 7 . In this paper we consider as the input signal $x$ an image with a resolution of $N_{1} \times N_{2}$ pixels, where $N_{1}$ and $N_{2}$ are positive even integers. Since the images we treat in this paper have appropriate periodic structure, we consider periodic boundary condition. In other word, MOGMRA is defined on the finite abelian group $\boldsymbol{Z} / N_{1} \boldsymbol{Z} \times \boldsymbol{Z} / N_{2} \boldsymbol{Z}$.

Then for example, at the second level, we have the 100 outputs (decomposed signals) by filtering by filters shown in Fig. 4. We denote these outputs as follows:

$$
\begin{array}{llllllll}
F_{7,0}^{2}(x) & G_{6,0}^{2}(x) & \cdots & G_{1,0}^{2}(x) & F_{0,0}^{2}(x) & & & \\
G_{7,1}^{2}(x) & A_{6,1}^{1,2}(x) & \cdots & A_{1,1}^{1,2}(x) & G_{0,1}^{2}(x) & A_{1,1}^{2,2}(x) & \cdots & A_{6,1}^{2,2}(x) \\
G_{7,2}^{2}(x) & A_{6,2}^{1,2}(x) & \cdots & A_{1,2}^{1,2}(x) & G_{0,2}^{2}(x) & A_{1,2}^{2,2}(x) & \cdots & A_{6,2}^{2,2}(x) \\
G_{7,3}^{2}(x) & A_{6,3}^{1,2}(x) & \cdots & A_{1,3}^{1,2}(x) & G_{0,3}^{2}(x) & A_{1,3}^{2,2}(x) & \cdots & A_{6,3}^{2,2}(x) \\
G_{7,4}^{2}(x) & A_{6,4}^{1,2}(x) & \cdots & A_{1,4}^{1,2}(x) & G_{0,4}^{2}(x) & A_{1,4}^{2,2}(x) & \cdots & A_{6,4}^{2,2}(x) \\
G_{7,5}^{2}(x) & A_{6,5}^{1,2}(x) & \cdots & A_{1,5}^{1,2}(x) & G_{0,5}^{2}(x) & A_{1,5}^{2,2}(x) & \cdots & A_{6,5}^{2,2}(x) \\
G_{7,6}^{2}(x) & A_{6,6}^{1,2}(x) & \cdots & A_{1,6}^{1,2}(x) & G_{0,6}^{2}(x) & A_{1,6}^{2,2}(x) & \cdots & A_{6,6}^{2,2}(x) \\
F_{7,7}^{2}(x) & G_{6,7}^{2}(x) & \cdots & G_{1,7}^{2}(x) & F_{0,7}^{2}(x) & & &
\end{array}
$$

Here the positions of the symbols are corresponding to the positions of filters in Fig. 4. For the $j$ th level, by the same way as in the second level, we denote the sig- 
Fig. 27 Distorted circle illusion (top). Green circles are apparently distorted, although they are concentric circles shown in the below. Concentric circles (bottom)
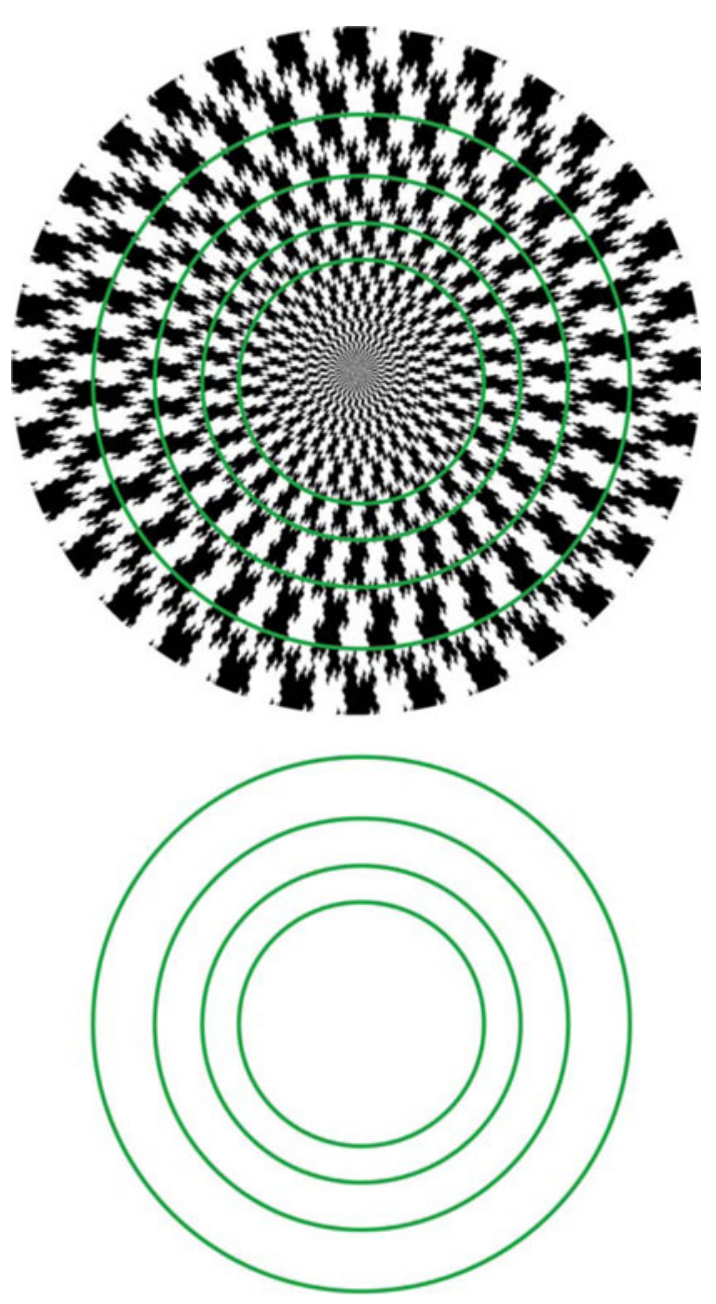

nals by $F_{k, l}^{j}(x), G_{k, l}^{j}(x), A_{k, l}^{1, j}(x), A_{k, l}^{2, j}(x)$. The signal $F_{0,0}^{j}(x)$ is called MOGMRA decomposed approximation of $x$ at the $j$ th level and others are called MOGMRA decomposed details of $x$ at the $j$ th level. For each decomposed signal we can gain the reconstructed signal by using filters for reconstruction. Since simple pinwheel framelets are tight frames, we can take as filters for reconstruction ones for decomposition [5]. We write the reconstruction signals from $F_{k, l}^{j}(x), G_{k, l}^{j}(x), A_{k, l}^{1, j}(x)$, and $A_{k, l}^{2, j}(x)$ by $\widetilde{F}_{k, l}^{j}(x), \widetilde{G}_{k, l}^{j}(x), \widetilde{A}_{k, l}^{1, j}(x)$, and $\widetilde{A}_{k, l}^{2, j}(x)$, respectively. The signal $\widetilde{F}_{0,0}^{j}(x)$ is MOGMRA reconstructed approximation at the $j$ th level, and others are MOGMRA reconstructed details at the $j$ th level. For details, see Section 3 in Arai and Arai [5]. Then for any positive integer $J$, the MOGMRA of simple pinwheel framelets have the perfect reconstruction property [5], that is, 
Fig. 28 a Unit of the fractal spiral illusion; $\mathbf{b}$ tiling of copies of the unit

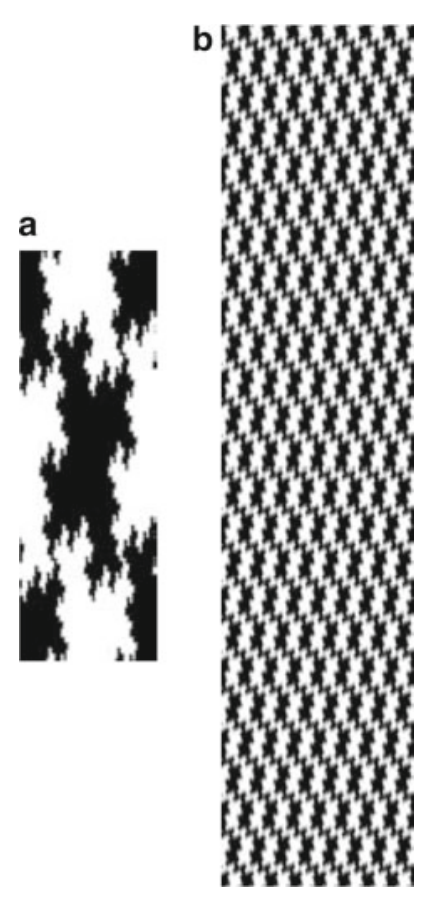

$$
\begin{aligned}
x= & \widetilde{F}_{0,0}^{J}(x)+\sum_{j=1}^{J}\left\{\sum_{(k, l) \in \Lambda_{f} \backslash\{(0,0)\}} \widetilde{F}_{k, l}^{j}(x)+\sum_{(k, l) \in \Lambda_{g}} \widetilde{G}_{k, l}^{j}(x)\right. \\
& \left.+\sum_{(k, l) \in \Lambda_{a}}\left(\widetilde{A}_{k, l}^{1, j}(x)+\widetilde{A}_{k, l}^{2, j}(x)\right)\right\},
\end{aligned}
$$

where

$$
\begin{aligned}
\Lambda_{f} & =\{(0,0),(0,7),(7,0),(7,7)\}, \\
\Lambda_{g} & =\{(k, l)\}_{k=0,7: l=1,2, \cdots, 6} \cup\{(k, l)\}_{l=0,7: k=1,2, \cdots, 6}, \\
\Lambda_{a} & =\{(k, l)\}_{k=1,2, \cdots, 6: l=1,2, \cdots, 6} .
\end{aligned}
$$

A precise description of general theory of MOGMRA is found in Arai and Arai [5] or Arai [2] (see also Arai and Arai [4]). In this paper, we use the case of $J=5$.

Computation of concentric circular filtering. Before describing our method, we will mention the structure of the fractal spiral figure. A preliminary step is to make the well-known fractal island. A recipe of the construction of the fractal island is found in [16]. It is known that the tiling of fractal islands can cover the whole plane. Next we consider a unit $X_{0}$ (Fig. 28a): the unit is a dilation of the fractal island, where the dilation ratio of height to width is $1: 3$. Then we consider the tiling $X_{1}$ of $8 \times 12$ 

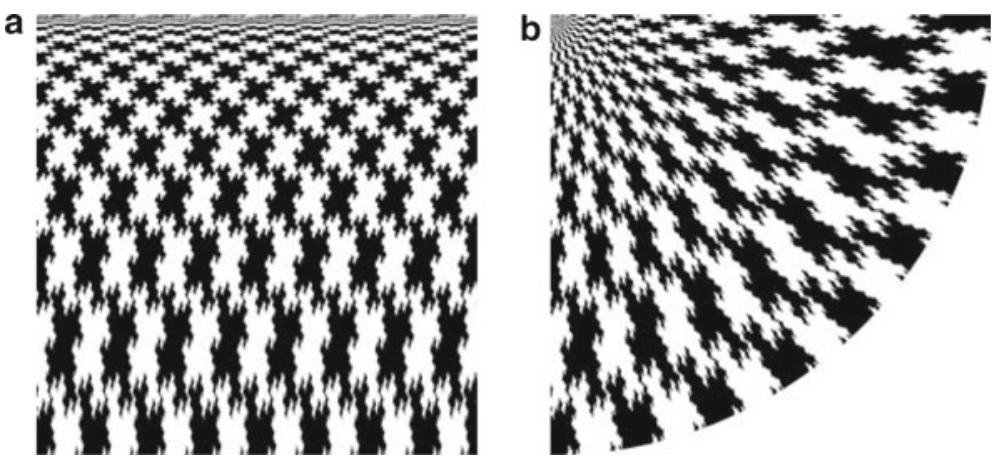

Fig. 29 a $X_{2} ; \mathbf{b} X_{3}$

units as shown in Fig. 28b (for saving space, the block is displayed in reduced size). In general, $f_{1}$ denotes the mapping which transforms a rectangle to a $8 \times 12$ tiling of copies of the rectangle (see Fig. 28). For example $f_{1}: X_{0} \rightarrow X_{1}$. Then we take the logarithmic scaling $X_{2}$ of $X_{1}$ (Fig. 28) as shown in Fig. 29a. Denote by $f_{2}$ the operation of such scaling. In particular, $f_{2}: X_{1} \rightarrow X_{2}$. Furthermore, we transform $X_{2}$ to $X_{3}$ by the conformal mapping which transforms Cartesian coordinate axes to polar coordinate axes (see Fig. 29b): Because of the periodic structure, firstly we consider only the quarter. We write this map by $f_{3}$, that is, we have $f_{3}: X_{2} \rightarrow X_{3}$. Rotating $X_{3}$ by anti-clockwise angles $\pi / 2, \pi$, and $3 \pi / 2$, we have $f_{41}\left(X_{3}\right), f_{42}\left(X_{3}\right)$, and $f_{43}\left(X_{3}\right)$. Lastly, let

$$
X_{4}=X_{3} \cup f_{41}\left(X_{3}\right) \cup f_{42}\left(X_{3}\right) \cup f_{43}\left(X_{3}\right) \text {. }
$$

Then $X_{4}$ is the fractal spiral figure (Fig. 2). We denote by $f_{4}$ the map transforming $X_{3}$ to $X_{4}$. Note that the map $f_{j}$ can be defined for all images with the same size as $X_{j-1}(j=1,2,3,4)$.

Concentric circular filtering is defined as follows: $R_{0}=f_{4} \circ f_{3} \circ f_{2}\left(\widetilde{F}_{0,0}^{5}\left(X_{1}\right)\right)$, and $R_{i}$ 's $(i \geqq 1)$ are a numbering of the signals $f_{4} \circ f_{3} \circ f_{2}\left(\widetilde{F}_{k, l}^{j}\left(X_{1}\right)\right)((k, l) \in$ $\left.\Lambda_{f} \backslash\{(0,0)\}\right), f_{4} \circ f_{3} \circ f_{2}\left(\widetilde{G}_{k, l}^{j}\left(X_{1}\right)\right)\left((k, l) \in \Lambda_{g}\right), f_{4} \circ f_{3} \circ f_{2}\left(\widetilde{A}_{k, l}^{1, j}\left(X_{1}\right)\right), f_{4} \circ f_{3} \circ$ $f_{2}\left(\widetilde{A}_{k, l}^{2, j}\left(X_{1}\right)\right)\left((k, l) \in \Lambda_{a}\right)$, where $j=1,2,3,4,5$.

Computation of hyperbolic filtering. The idea of computation of hyperbolic filtering is almost same as the concentric circular filtering. However in hyperbolic case we exclude 1:3 scaling and the process of logarithmic scaling. Let $Y_{0}$ be the unit of the illusion of fringed edges (Fig. 30a).

Let $g_{1}$ be the operator to make the tiling of $8 \times 8$ units (see Fig. 30a), and let $Y_{1}=g_{1}\left(Y_{0}\right)$. Let $g_{2}$ be the conformal mapping which transforms Cartesian coordinate axes to rectangular hyperbolic coordinate axes, and let $Y_{2}=g_{2}\left(Y_{1}\right)$ (see Fig. 30): Because of the periodic structure, firstly only the quarter is considered. Rotating $Y_{2}$ by anti-clockwise angles $\pi / 2, \pi$, and $3 \pi / 2$, we have $g_{31}\left(Y_{2}\right), g_{32}\left(Y_{2}\right)$, and $g_{33}\left(Y_{2}\right)$. Lastly, let

$$
Y_{3}=Y_{2} \cup g_{31}\left(Y_{2}\right) \cup g_{32}\left(Y_{2}\right) \cup g_{33}\left(Y_{2}\right) .
$$



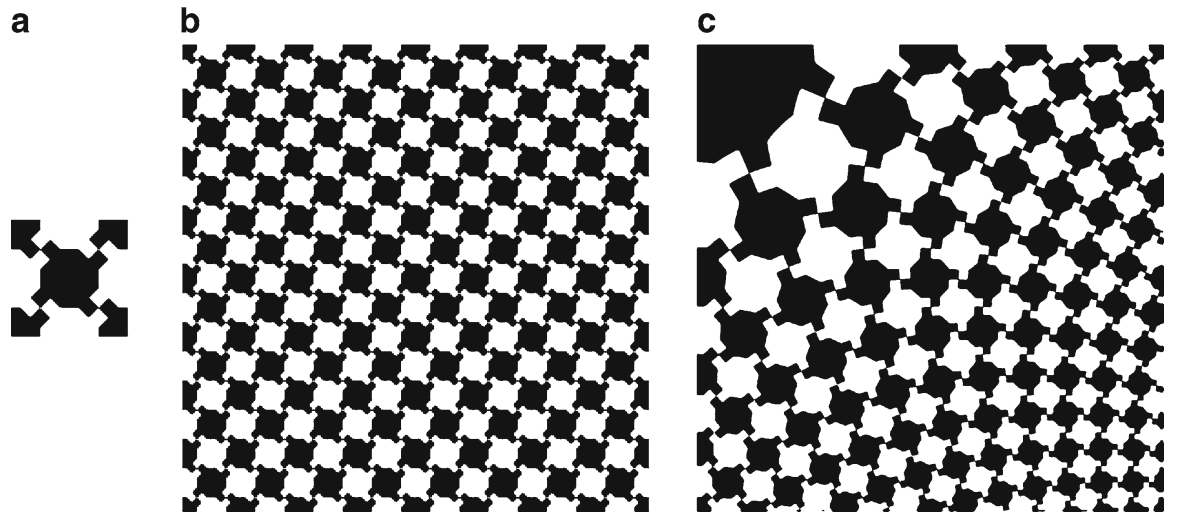

Fig. 30 a Unit of illusion of fringed edges [15]; b block of the units (see Kitaoka [14]); c hyperbolic deformation of $\mathbf{b}$

Let $g_{3}$ denote the map transforming $Y_{2}$ to $Y_{3}$. Note that the maps $g_{j}$ can be defined for all images with the same size as $Y_{j-1}(j=2,3)$. Let $R_{0}^{\prime}=g_{3} \circ g_{2}\left(\widetilde{F}_{0,0}^{5}\left(Y_{1}\right)\right)$, and $R_{i}^{\prime}$ 's $(i \geqq 1)$ a numbering of the signals $g_{3} \circ g_{2}\left(\widetilde{F}_{k, l}^{j}\left(Y_{1}\right)\right)\left((k, l) \in \Lambda_{f} \backslash\{(0,0)\}\right)$, $g_{3} \circ g_{2}\left(\widetilde{G}_{k, l}^{j}\left(Y_{1}\right)\right)\left((k, l) \in \Lambda_{g}\right), g_{3} \circ g_{2}\left(\widetilde{A}_{k, l}^{1, j}\left(Y_{1}\right)\right), g_{3} \circ g_{2}\left(\widetilde{A}_{k, l}^{2, j}\left(Y_{1}\right)\right)\left((k, l) \in \Lambda_{a}\right)$, where $j=1,2,3,4,5$.

\section{Further problems}

In this paper we classified CMRA reconstructed details for the fractal spiral illusion into four classes stated in Sect. 2, and HMRA reconstructed details for the hyperbolic illusion of fringed edges into seven classes in Sect. 3. These operations are based on our personal cognition. However the authors think that the results are correct, that is, as figures in this paper indicate, we have been able to control accurately illusory components of the fractal spiral illusion and of the hyperbolic illusion of fringed edges. A crucial problem is to find a mathematical algorithm of grouping reconstructed details. However this problem seems to be difficult, because the definitions of the classes are essentially whether one can perceive a designated illusion on a signal or not. Therefore the desired algorithm is nothing other than an algorithm of our cognition.

In Sect. 4 we reported two phenomena related to the fractal spiral illusion. In order to resolve the problem why two phenomena occur, we need to study further mathematical theory of color perception and mathematical models of visual information processing of extrastriate cortex.

Remark 1 The fractal spiral illusion was found in our unpublished paper, H. Arai and S. Arai, "On a spiral illusion generated by a fractal set" (in Japanese), Mathematical Vision Science Lab. Technical Report 3 (2007) (available from URL: http://www4. ocn.ne.jp/ arai/mvel/e-repo003.pdf). 
Acknowledgments The authors thank Professor Akiyoshi Kitaoka for his valuable suggestion, and for naming our spiral illusion (Fig.2) the fractal spiral illusion.

\section{References}

1. Arai, H.: A nonlinear model of visual information processing based on discrete maximal overlap wavelet. Interdiscip. Inf. Sci. 11, 177-190 (2005)

2. Arai, H.: Wavelets. Kyoritsu Publ. Co., Tokyo (2010) (in Japanese)

3. Arai, H., Arai, S.: Common factor of a certain kind of tilt illusions clarified by a wavelet, VISION. J. Vis. Soc. Japan 17, 259-265 (2005) (in Japanese)

4. Arai, H., Arai, S.: Finite discrete, shift-invariant, directional filterbanks for visual information processing, I: Construction. Interdiscip. Inf. Sci. 13, 255-273 (2007)

5. Arai, H., Arai, S.: 2D tight framelets with orientation selectivity suggested by vision science. Invited paper. JSIAM Lett. 1, 9-12 (2009)

6. Cohen, A., Daubechies, I., Feauveau, J.-C.: Biorthogonal bases of compactly supported wavelets. Commun. Pure Appl. Math. 45, 485-560 (1992)

7. Coifman, R.R., Donoho, D.L.: Translation invariant de-noising. Lect. Notes Stat. 103, 125-150 (1995)

8. Daubechies, I., Han, B., Ron, A., Shen, Z.: Framelets: MRA-based construction of wavelet frames. Appl. Comput. Harmon. Anal. 14, 1-46 (2003)

9. Fraser, J.: A new visual illusion of direction. Br. J. Psychol. 2, 307-320 (1908)

10. Gallant, J.L., Braun, J., Van Essen, D.C.: Selectivity for polar, hyperbolic, and Cartesian gratings in macaque visual cortex. Science 259, 100-103 (1993)

11. Gallant, J.L., Conner, C.E., Rakshit, S., Lewis, J.W., Van Essen, D.C.: Neural responses to polar, hyperbolic, and Cartesian gratings in area V4 of the macaque monkey. J. Neurophysiol. 76, 27182739 (1996)

12. Gregory, R.L., Heard, P.: Border locking and the Cafe Wall illusion. Perception 8, 365-380 (1979)

13. Kitaoka, A.: Tilt illusions after Oyama (1960): a review. Jpn. Psychol. Res. 49, 7-19 (2007)

14. Kitaoka, A.: Geometrical illusions. In: Goto, T., Tanaka, H. (eds.) Handbook of the Science of Illusion, pp. 56-77. University of Tokyo Press, Tokyo (2005) (in Japanese)

15. Kitaoka, A., Pinna, B., Brelstaff, G.: New variants of the spiral illusion. Perception 30, 637-646 (2001)

16. Lauwerier, H.A.: Fractals: Endlessly Repeated Geometrical Figures. Princeton University Press, Princeton (1991)

17. Morgan, M.J., Moulden, B.: The Münsterberg figure and twisted cords. Vis. Res. 26, 1793-1800 (1986)

18. Nason, G.P., Silverman, B.W.: The stationary wavelet transform and some statistical applications. Lect. Notes Stat. 103, 281-299 (1995)

19. Percival, D.B., Walden, A.T.: Wavelet Methods for Time Series Analysis. Cambridge University Press, Cambridge (2000) 